\title{
Sulfation of Lithocholate as a Possible Modifier of Chenodeoxycholic Acid-induced Elevations of Serum Transaminase in Patients with Gallstones
}

\author{
J. W. Marks, S. O. Sue, B. J. Pearlman, G. G. Bonorrus, P. Varady, \\ J. M. LACHIN, and L. J. SCHOENFIELD, Division of Gastroenterology, \\ Department of Medicine, Cedars-Sinai Medical Center, Los Angeles, \\ California 90048; University of California at Los Angeles School \\ of Medicine, Los Angeles, California 90048, and Department of Biostatistics, \\ George Washington University, Bethesda, Maryland 20014
}

\begin{abstract}
A B S T RA C T Chenodeoxycholic acid (CDC), through its metabolite, lithocholic acid (LC), is hepatotoxic in certain species. The cause of elevations of serum transaminase in $25 \%$ of humans ingesting CDC, however, is unknown, but also may be due to LC. Because efficient hepatic sulfation of LC may protect against hepatic injury, the aim of this study was to determine if sulfation of LC might modify CDCinduced elevations of transaminase. Pretreatment sulfation fraction (SF) was estimated in 63 randomly selected patients with gallstones in a double-blind randomized trial of CDC, $750 \mathrm{mg} / \mathrm{d}, 375 \mathrm{mg} / \mathrm{d}$, or placebo; in 27 of these, SF was repeated at 1 or $2 \mathrm{yr}$. In four other patients, the SF was measured at $2 \mathrm{yr}$ only. Serum glutamic oxaloacetic transaminase and serum glutamic pyruvic transaminase were determined monthly for $3 \mathrm{mo}$ and then every 3 or $4 \mathrm{mo}$; an elevation of transaminase was defined as $>150 \%$ of the normal upper limit in asymptomatic patients. $10 \mu \mathrm{Ci}$ of ${ }^{3} \mathrm{H}$ glyco-LC (sp act $84 \mathrm{mCi} / \mathrm{mol}$ ) was ingested $10-12 \mathrm{~h}$ before fasting duodenal biliary drainage. Bile acids in bile were separated by thin-layer chromatography. The SF was estimated as a percentage of total radioactivity (scintillation counting) in sulfated glyco-LC. The standard deviation for replicate SF determinations $(n=311)$ was $2.1 \%$ The pretreatment SF (mean $60.7 \pm 1.7$ SEM) correlated inversely, with age $(r$ $=0.336, P<0.005)$ and directly with the obesity index $(r=0.495, P>0.001)$, but was independent of
\end{abstract}

This work was presented in part at the Annual Meeting of the American Gastroenterology Association, Salt Lake City, Utah, May 1980, and the VI International Bile Acid Meeting, Freiburg, West Germany, October 1980.

Received for publication 3 December 1980 and in revised form 16 July 1981. sex. The SF, remeasured at 1 or $2 \mathrm{yr}$, did not change significantly with time or CDC. Among CDC-treated patients, elevations of transaminase occurred in $75 \%$ of patients with a SF $<45 \%$ vs. $11 \%$ with a SF $>45 \%$ $(P<0.001)$. In conclusion, a $\mathrm{SF}<45 \%$ occurred in patients with gallstones who had a high probability of developing elevated serum transaminase when treated with CDC. Thus, sulfation of lithocholate may modify CDC-induced elevations of serum transaminase.

\section{INTRODUCTION}

Chenodeoxycholic acid (CDC) ${ }^{1}$ administered orally dissolves gallstones in humans. Approximately 25\% of patients receiving CDC develop transient elevations of the serum transaminase, but other hepatic chemistries usually are normal and hepatic morphology is not different from that of untreated patients with gallstones $(1,2)$. The pathogenesis and clinical significance of these CDC-induced elevations of transaminase remain to be determined.

CDC is converted in humans by intestinal microorganisms to lithocholic acid (LC) (3). Dose-related biochemical and morphological hepatotoxicity of CDC has been demonstrated in Rhesus monkeys $(4,5)$ and baboons (6). The hepatoxicity of CDC appears to be due to hepatobiliary accumulations of LC $(7,8)$. Hepatic sulfation of LC produces a more excretable form of LC and thereby is a major determinant of

\footnotetext{
${ }^{1}$ Abbreviations used in this paper: CDC, chenodeoxycholic acid; LC, lithocholic acid; NCGS, National Cooperative Gallstone Study; SF, sulfation fraction; SGOT, serum glutamic oxaloacetic transaminase; SGPT, serum glutamic pyruvic transaminase.
} 
hepatobiliary levels of LC $(9,10)$. Sulfated LC also may be less toxic than nonsulfated LC $(7,11,12)$. Species that sulfate LC poorly, e.g., Rhesus monkeys (13) and baboons (14), manifest CDC-induced elevations of biliary LC and hepatotoxicity. Species that sulfate LC efficiently, e.g., chimpanzees (12) and humans (15), may be protected from toxic accumulations of LC.

Most humans sulfate LC efficiently (15). Stiehl et al. (16), however, reported a wide variation among individuals in the percentage of sulfated LC in bile (20-90\% of total LC). Sulfation of LC, assessed by administration of radiolabeled $L C$, is a reproducible measurement within individuals even during treatment with CDC $(17,18)$. The suggestion has been made, therefore, that the ability of sulfate LC may be a genetically determined characteristic (19). Moreover, individuals with an impaired ability to sulfate LC might be analogous metabolically to species of animals that sulfate LC poorly and are susceptible to CDC-induced hepatic abnormalities. Accordingly, the aim of this study was to determine whether the degree of sulfation of administered radiolabeled LC was a modifier of CDC-induced elevations of serum transaminase.

\section{METHODS}

Experimental design. The patients were participants in the National Cooperative Gallstone Study (NCGS), a multicenter, double-masked, clinical trial of randomly allocated oral CDC ( 750 or $375 \mathrm{mg} / \mathrm{d}$ ) vs. placebo for $2 \mathrm{yr}$ for the dissolution of gallstones in 1,000 patients (20). Potential hepatotoxic agents and antihyperlipidemic or other drugs known to influence the composition of lipids were proscribed. 67 of the 142 patients, 25 receiving $750 \mathrm{mg} / \mathrm{d}$ CDC, 17 receiving $375 \mathrm{mg} / \mathrm{d}$ CDC, and 25 receiving placebo at Cedars-Sinai Medical Center ( 1 of the 10 centers in the NCGS), underwent a total of 98 studies of sulfation with informed consent according to the principles of the Declaration of Helsinki.

Studies of sulfation were done in conjunction with cholecystokinin-stimulated duodenal biliary drainage, which was performed as a routine procedure in the NCGS with the patient fasting before treatment and at the month 12 and 24 visits. The patients had not received radiographic contrast material or BSP within $72 \mathrm{~h}$ preceding the duodenal drainage. Studies of sulfation were done before treatment in 63 patients; before treatment and at $1 \mathrm{yr}$ in 22 patients, four of whom had a third study at $2 \mathrm{yr}$; before treatment and at $2 \mathrm{yr}$ in five patients; and only at $2 \mathrm{yr}$ in four patients. Patients who had studies done before treatment and at 1 yr were selected according to a scheme for randomization; the studies done at $2 \mathrm{yr}$ were done in all available patients who agreed to participate. Thus, 63 studies were done before treatment, 22 at $1 \mathrm{yr}$ and 13 at 2 yr (Table I).

Serum glutamic oxaloacetic (SGOT) and pyruvic (SGPT) transaminases were determined before treatment, monthly the first $3 \mathrm{mo}$, every 3 mo the remainder of yr 1 , and every $4 \mathrm{mo}$, yr 2. The transaminases were measured independently by the clinical laboratory at Cedars-Sinai and by the central serum laboratory of the NCGS (Bio-Science, Van Nuys, Calif.). A CDC-induced abnormality of serum transaminase was predefined by the NCGS $(20)$ as a confirmed (immediately repeated) elevation $\geq 150 \%$ of the upper limit of
TABLE I

Experimental Design: Numbers of Patients Studied and Sulfation Fractions Determined

\begin{tabular}{crcc}
\hline & \multicolumn{3}{c}{ Sulfation fractions* } \\
\cline { 2 - 4 } Number of patients & Pre Rx & $1 \mathrm{yr}$ & $2 \mathrm{yr}$ \\
\hline 36 & 36 & - & - \\
18 & 18 & 18 & - \\
4 & 4 & 4 & 4 \\
5 & 5 & - & 5 \\
4 & - & - & 4 \\
67 & 63 & 22 & 13 \\
\hline
\end{tabular}

$* n=98$.

normal at either of the testing facilities. Elevations of serum transaminase associated with biliary pain were excluded to eliminate gallstone-induced elevations. Biliary pain was defined as a steady pain of moderate or severe intensity, usually located in the upper abdomen, and lasting $>15$ min. Elevations of serum transaminase were followed by repeating the determinations every 2 wk until resolution.

The studies of sulfation were done without knowledge of the treatment assignments of patients or the results of the determinations of serum transaminase or biliary lipids or bile acids. The results were analyzed statistically using $t$ tests and analysis of variance.

Lithocholic acid sulfation fraction. The sulfation fraction (SF) was defined as the percentage of recovered radiolabeled LC in the bile as sulfated LC. $10 \mu \mathrm{Ci}$ of glycolithocholic acid (lithocholic- ${ }^{3} \mathrm{H}(\mathrm{G})$ sp act $84 \mathrm{mCi} / \mathrm{mmol}$, New England Nuclear, Boston, Mass.) in cherry syrup (Parke-Davis, Div. of Warner-Lambert Company, Morris Plains, N.J.) was administered orally at 10:00 p.m. the night before the patient was scheduled for biliary drainage.

Duodenal intubation was performed between 8:00 and 10:00 a.m. after a 12-16 h overnight fast. A single lumen tube was positioned in the third part of the duodenum under fluoroscopic control, and the duodenum was flushed with $\mathbf{5 0}$ $\mathrm{ml}$ of $37^{\circ} \mathrm{C}$ normal saline. Contraction of the gallbladder then was stimulated by intravenous cholecystokinin, 75 Ivy units in $10 \mathrm{ml}$ saline administered over $1-2 \mathrm{~min}$. A $20-40-\mathrm{ml}$ sample of concentrated bile was obtained, mixed thoroughly, and maintained at $37^{\circ} \mathrm{C}$.

A $1.0-\mathrm{cm}^{3}$ aliquot of the collected bile was placed in $19 \mathrm{~cm}^{3}$ 95\% ethyl alcohol and the cholesterol was extracted three times with petroleum ether. The alcohol phase containing the bile acids was dried in vacuo and reconstituted in $2-3 \mathrm{~cm}^{3}$ methanol. No loss of isotope occurred during this extraction.

Thin-layer chromatography was performed using silica Gel H plates (Brinkmann Instruments, Inc., Westbury, N. Y.) and two solvent systems: Hofmann's (21) S-VIII n-propanol/ proprionic acid-isoamylacetate/water 10:15:20:5 (vol/vol) and Palmer's (20) Butanol $3 \mathrm{~N}$-butanol/0.01 M Tris buffer/ proprionic acid [50:9.25:0.75 (vol/vol)]. An aliquot of the methanol solution containing a known amount of radioactivity was applied to the origin of the precoated plate along with LC standards. The sulfolithocholylglycine standard was synthesized from glycolithocholic acid (98\% pure, Calbiochem-Behring Corp., San Diego, Calif.), according to the method of Fieser (22). The sulfate chromatographed as a single spot without tailing and was separated from glycolithocholic acid by a retardation factor of $0.20-0.25$ in either solvent system (Fig. 1). 


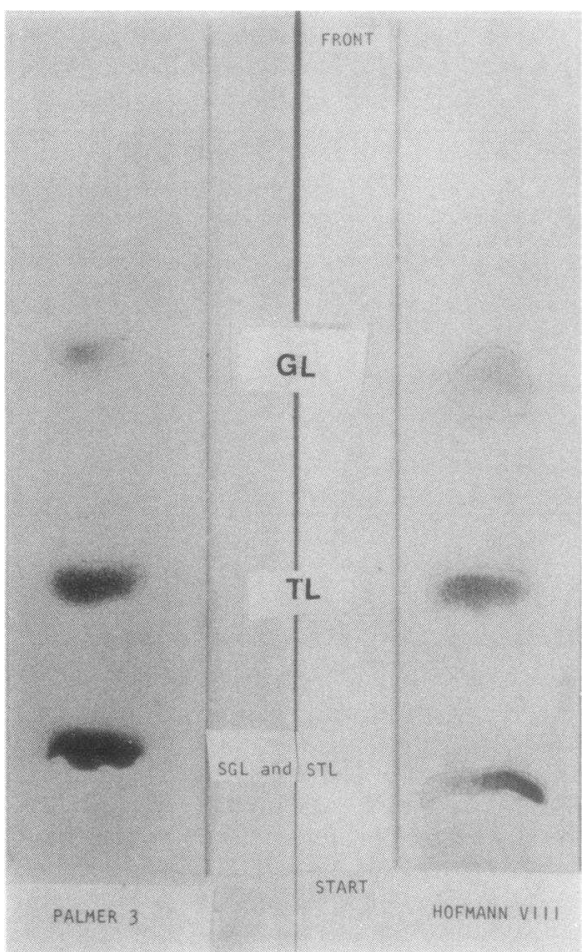

Figure 1 Thin-layer chromatography of sulfated glycolithocholate (SGL), sulfated taurolithocholate (STL), glycolithocholate (GL), and taurolithocholate (TL). Palmer 3 and Hofmann VIII refer to the solvent systems described in the text.

The area on the plate containing the standards was sprayed with $10 \%$ phosphomolybdic acid and the equivalent areas containing glycolithocholic acid and sulfolithocholylglycine were scraped into separate counting vials. Water $\left(0.5 \mathrm{~cm}^{3}\right)$ and Aquasol $\left(10 \mathrm{~cm}^{3}\right)$ were added, and the radioactivity was measured using a Searle Mark III liquid scintillation counter (Searle Analytic, Inc., Des Plaines, Ill.) with external standardization and automatic quench correction.

The SF, expressed as a percentage, was calculated as the ratio of the amount of radioactivity in the area of sulfolithocholylglycine to the amount of radioactivity applied to the origin $\times 100$. Virtually all radioactivity applied to the plate was accounted for by the counts at the origin $(4.8 \% \pm 0.4)$ and in the areas of sulfolithocholylglycine and glycolithocholic acid. All determinations on bile specimens were done at least in duplicate; these replicate samples (up to six, per specimen, $n=311$ ) exhibited a standard deviation of $2.1 \%$.

Analyses of biliary lipids. Bile was analyzed for biliary lipid and bile acid composition at the Central Bile Laboratory of the NCGS (La Jolla, Calif., Dr. Scott Grundy, Director) in order to maintain the mask of the study. Total and individual bile acids, lecithin, and cholesterol were determined by standardized methods described elsewhere (23). All samples containing $2 \%$ or greater LC were analyzed further at the central laboratory for the percentage of sulfated LC (24). The cholesterol saturation index was calculated for each sample of bile according to the criteria of Hegardt and Dam (25) using the Thomas-Hofmann equation (26). Procedures for external quality control, based on masked analyses of split duplicates, revealed that the coefficients of variation were $14 \%$ for the cholesterol saturation index, $8 \%$ for CDC, and
$49 \%$ for total LC. Procedures for internal quality control revealed a coefficient of variation of $23 \%$ for sulfated LC, but external quality control was not available.

\section{RESULTS}

Sulfation Fractions. Fig. 2 presents a distribution histogram of the values for SF determined in the 63 patients at base line. The mean SF was $60.7 \pm 1.7 \mathrm{SEM}$ (range, 27-89\%). A break in the distribution of values was observed at $45 \%$. Patients with values $<45 \%$ were deemed inefficient sulfators relative to patients with values $>45 \%$. A statistical test for normal distribution (Statistical Analysis System Institute Program, 1979) demonstrated a significant $(P<0.01)$ deviation from normality among all patients due to the negative skewness evident in Fig. 2, suggesting that the inefficient sulfators comprised a separate group, recognizing the limits of the small sample size.

Significantly more of the inefficient sulfators than efficient sulfators were married $(P<0.05)$ or less than ideal body weight $(P<0.001)$. No other of the 24 demographic, clinical, or biochemical characteristics tabulated at base line (20) were significantly more or less prevalent among the inefficient sulfators. With or without inclusion of the inefficient sulfators, the SF tended to decline with increasing age $(r=-0.336$, $P<0.005)$ and increase with increasing obesity index $(r=0.495, P<0.001)$, but did not correlate with sex. The biological significance of the statistical relationship between the SF and the age or obesity index, however, is questionable because of the scatter of the data. Also, no significant correlation was found among the inefficient sufators between the SF and the age or obesity index, again recognizing the limits of the small sample size.

Although the values for SF varied considerably among patients, values in individuals tended to re-

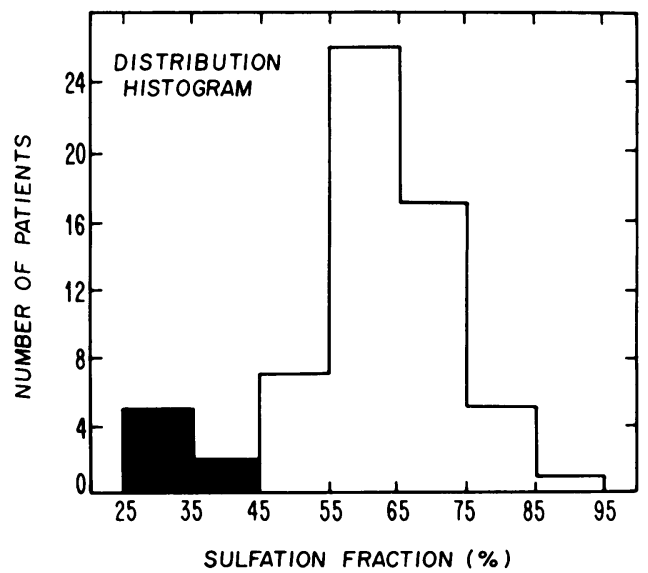

FIGURE 2 Distribution histogram of the values for sulfation fractions determined at base line in 63 patients. 
REPEAT SULFATION FRACTION

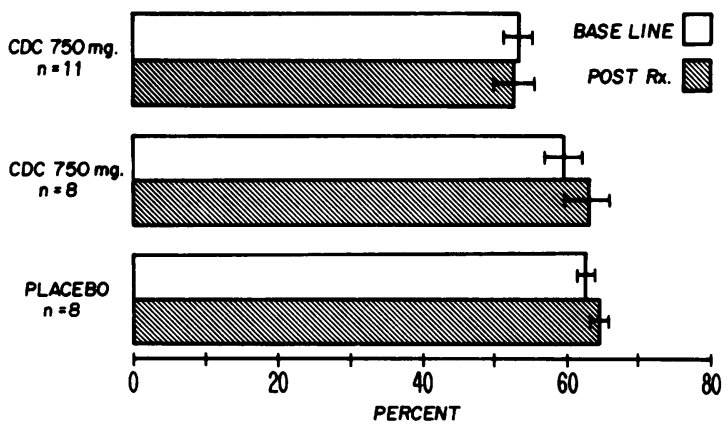

FIGURE 3 Sulfation fractions by treatment groups at base line and repeated at 1 or $2 \mathrm{yr}$ (posttreatment).

main constant. Fig. 3 shows the SF at base line and repeated at 1 or $2 \mathrm{yr}$ in 27 patients according to their treatment assignment. The base-line or posttreatment values did not differ significantly among the treatment groups. Also, within each treatment group, no significant difference was found between base-line and posttreatment SF. Although the statistical power for detecting differences was limited by the relatively small numbers of patients in each group, values in the same patient always were either above or below $45 \%$.

Sulfation fractions and elevations of serum transaminase. 11 of 67 patients developed elevations of the serum transaminase without biliary pain; of these 11 patients, 9 had received $750 \mathrm{mg}$ CDC/d, 1 had received $375 \mathrm{mg} / \mathrm{d}$, and 1 had received placebo
(Table II). All other hepatic chemistries remained normal (20). Among the 11 patients, the percentage of whom were women $(45 \%)$ and the mean age $(62.2$ $\mathrm{yr} \pm 2.8)$ were not significantly different from the other 56 patients $(52 \%$ women and $59.0 \mathrm{yr} \pm 1.3)$, but the obesity index $(1.048 \pm 0.04$ vs. $1.153 \pm 0.03, P<0.05)$ and the SF $(45.8 \% \pm 5.2$ vs. $62.4 \% \pm 1.5, P<0.01)$ were significantly lower. Patients who developed elevations of the serum transaminase had significantly higher $(P<0.01)$ levels at base line of the SGOT $(34.3 \pm 1.5$ vs. $28.5 \pm 0.8)$ and SGPT $(32.7 \pm 3.4$ vs. $23.7 \pm 1.1)$ than those who did not develop elevations of the serum transaminase. No other of the 24 demographic, clinical, or biochemical characteristics tabulated at base line (20) were significantly more or less prevalent among the patients who developed elevations of the serum transaminase than among those who did not. The elevations of serum transaminase returned to normal with continued therapy in all patients except patient 9 in whom the elevation reverted to normal only when treatment was discontinued, at which time an hepatic biopsy was performed and interpreted as showing normal morphology.

Fig. 4 shows the percentage of patients developing elevations of serum transaminase classified according to whether their SF was more or less than $45 \%$ at base line or during treatment. Patients who received placebo were not included in this figure because only those patients treated with CDC were at risk for the development of drug-related elevations of serum transaminase. Elevations of transaminase occurred

TABLE II

Patients Having Elevations of the Serum Transaminase

\begin{tabular}{ccccccccc}
\hline & & & & & & \multicolumn{3}{c}{ Transaminase elevation } \\
\cline { 6 - 8 } Patient & CDC & Sex & Age & $\begin{array}{c}\text { Obesity } \\
\text { index }\end{array}$ & $\begin{array}{c}\text { Sulfation } \\
\text { fraction }\end{array}$ & Onset & Highest level & Duration \\
\hline & $m g / d$ & & $y r$ & & & mo & \% upper nl limit & mo \\
1 & 750 & F & 60 & 1.077 & 27 & 12 & 300 & 1 \\
2 & 750 & M & 54 & 1.073 & 39 & 9 & 150 & 1 \\
3 & 750 & M & 68 & 0.877 & 28 & 2 & 150 & 3 \\
4 & Placebo & F & 72 & 0.850 & 31 & 6 & 200 & 1 \\
5 & 375 & M & 58 & 0.901 & 33 & 5 & 300 & 3 \\
6 & 750 & F & 69 & 1.084 & 39 & 16 & 150 & 2 \\
7 & 750 & M & 77 & 0.886 & 41 & 2 & 300 & 0.5 \\
8 & 750 & F & 57 & 1.189 & 59 & 2 & 300 & 2 \\
9 & 750 & M & 49 & 1.150 & 68 & 3 & 600 & 3 \\
10 & 750 & M & 51 & 1.143 & 68 & 1 & 150 & 1 \\
11 & 750 & F & 69 & 1.300 & 71 & 1 & 300 & 1 \\
Mean & & & 62.2 & 1.048 & 45.8 & 5.4 & 264 & 1.7 \\
Standard & & & & & & & & \\
error & & & 2.8 & 0.04 & 5.2 & 1.5 & 44 & 0.3 \\
\hline
\end{tabular}

* The SF determined at base line is presented except for patients 1 and 2 in whom the $\mathrm{SF}$ was determined at $2 \mathrm{yr}$ only. 
SULFATION FRACTION, CDC PATIENTS ONLY
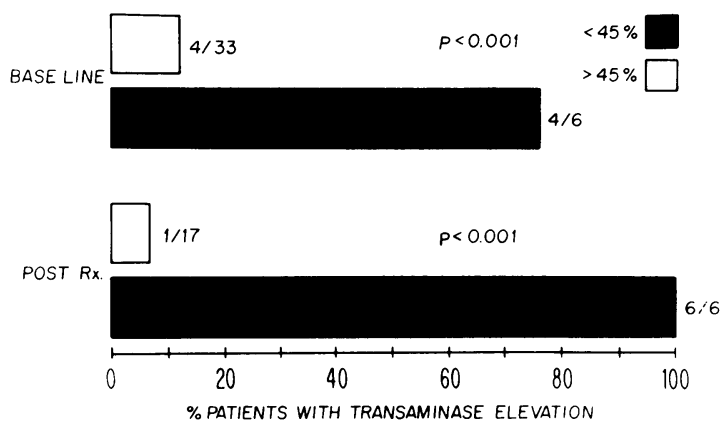

Figure 4 Elevations of serum transaminase in CDC-treated patients in relation to their sulfation fractions (SF) determined at base line and at 1 or 2 yr (posttreatment). All but 2 of the 6 patients with SF $<45 \%$ and 2 of the 17 patients with SF $>45 \%$ posttreatment also had SF determined at base line.

more frequently among the inefficient sulfators, i.e., with $\mathrm{SF}<45 \%$, both at base line and during treatment (each $P<0.001$ ). Among patients treated with CDC, $6(75 \%)$ of 8 patients with SF $<45 \%$ and only $4(11 \%)$ of 35 patients with $\mathrm{SF}>45 \%$ had elevations of serum transaminase $(P<0.001)$.

Biliary lipids. At the central bile laboratory, sulfated LC in bile was determined only in patients with a total LC $>2 \%$. Accordingly, sulfated LC was measured directly in the same bile sample in only 14 (6 at base line and 8 posttreatment) of our patients. A significant correlation was found between the SF and the percent sulfated LC. $(r=0.6603, P<0.005)$; however, only one of these patients had an $\mathrm{SF}<45 \%$ and one other patient (with an SF of 70\%) had an elevation of serum transaminase.

No significant correlation was found between the SF and the proportions of any of the individual bile acids or the cholesterol saturation index in bile. Because sulfation may protect by enhancing excretion of LC, inefficient sulfators might be expected to have a higher ratio of LC/CDC in bile, but this was not found. Furthermore, no significant difference was found in the LC/CDC ratio between patients with vs. those without elevations of the serum transaminase. No significant correlation was found between the proportion of total LC or sulfated LC in bile and the SGOT or SGPT determined simultaneously at base line and at 12 and 24 mo.

\section{DISCUSSION}

Method for determination of sulfation fraction. The SF was calculated by determining the proportion of labeled glycolithocholate that was sulfated, so that variations in the recovery of the total label in bile did not influence the results. Because as much as $50 \%$ of nocturnal hepatic biliary secretion may bypass storage in the gallbladder, and the degree of enterohepatic cycling may vary in individuals, the SF actually may be the result of one or more hepatic clearances (27), and therefore different absorption rates for LC and sulfated LC might affect measurement of SF. Nevertheless, determinations of the SF in individual patients were reproducible at 1- or 2-yr intervals.

We did not study diurnal variations in the formation, absorption, or metabolism of LC, but Allan et al. (15) found no difference in the proportion of biliary LC between early morning and late afternoon samples of bile from healthy controls or patients with gallstones. Theoretically, the SF also could be affected by desulfation of sulfated LC in the enterohepatic system. Although we did not measure desulfation, others have found that this occurs to a minimal degree (9). The predominant site of sulfation appears to be the liver (10), but sulfotransferase activity also has been detected in the intestinal mucosa (28). The influence of extrahepatic sulfation of LC on the SF remains to be determined. The $\mathrm{SF}$ was a reproducible measurement, despite the possibility that an unknown consumption of alcohol or viral illness might have affected the results. No significant difference was found in the reported amounts of alcohol consumed between the efficient and inefficient sulfators. Finally, our patients were not known to be ingesting drugs that are metabolized primarily by sulfation.

Previous determinations of sulfated lithocholate. The values for SF we found are comparable to data reported by previous investigators who measured the biotransformation of radiolabeled LC in humans. Cowen et al. (18) demonstrated that $60 \%$ of an intravenously administered dose of radiolabeled LC was sulfated in healthy subjects. Palmer and Bolt (11) found 41 and $75 \%$ sulfation in two patients with cholelithiasis studied in a similar manner. Other investigators have shown, as we did, that CDC does not significantly affect the sulfation of LC. Allan et al. (15) found equivalent degrees of sulfation of LC in patients with gallstones as in healthy subjects. These same investigators also reported no difference in sulfated LC levels in the serum of patients with gallstones before and after ingestion of CDC for $6 \mathrm{mo}$ (17). However, because the number of patients undergoing our study during CDC therapy was small, an effect of CDC therapy on SF cannot be excluded with certainty.

The range of values reported for the direct measurement of the percent sulfated LC in bile $(16,29)$ is comparable to the values for SF that we found after administration of labeled LC. In contrast to the agreement found in the biotransformation studies noted above, however, direct measurements of sulfated LC have yielded conflicting results in the literature with regard to the effect of CDC. Bremmelgaard et al. (29) found no change in the proportion of sulfated bile acids in patients with gallstones before and after they ingested CDC for 3-4 mo. In contrast, Stiehl and co-workers 
(30) reported that sulfated LC increased from a base line of 32.8 to $73.9 \%$ after 8 wk of CDC. Determination of the SF after a shorter period of CDC treatment in our study might have shown an induction effect or determinations by Stiehl and co-workers (30) after a longer period might have eliminated the apparent induction. In our study sufficient data were not obtained on the direct measurement of the percent sulfated LC in the bile of patients who had low values for the SF or who developed elevations of the serum transaminase.

Elevations of transaminase. The cause and clinical significance of the dose-related elevations of serum transaminase in CDC-treated patients with gallstones remains to be determined $(1,31)$. Our data are in accord with the hypothesis that CDC induces hepatic injury through its conversion to LC. The hypothesis is that among inefficient sulfators, unsulfated LC derived from CDC causes the elevations of the serum transaminase which signify acute injury to the liver. Whether any of these subjects will develop chronic hepatic disease remains to be determined.

Sulfation may render LC less toxic by facilitating its excretion and reducing its hepatotoxicity $(7,11$, 32-34). Sulfolithocholylglycine, however, has solubility characteristics that suggest potential hepatotoxicity (35). In fact, a recent study has shown that glycolithocholic acid sulfate, but not lithocholic acid sulfate or taurolithocholic acid sulfate causes cholestasis in rats (36).

A low SF may predispose to elevations of the serum transaminase in patients treated with CDC. It is possible, however, that inefficient sulfation is not the cause of the elevations of transaminase because both the low SF and hypertransaminasemia could result from an inapparent hepatic disease or a metabolic derangement. For example, patients who developed elevations of the serum transaminase had higher levels of serum transaminase within the normal range at base line. Furthermore, some individuals might have enhanced bacterial conversion of CDC to LC, resulting in increased LC absorption that exceeds the capacity of the liver to sulfate.

If inefficient sulfation persists, then persistent (rather than transient) elevations of the serum transaminase might be expected, but have been observed only occasionally $(1,31)$. Although the SF remained constant, it is possible that greater differences might have been seen if the SF had been determined also at the time of elevations of the transaminase. An adaptive mechanism could be postulated to explain the transient elevations of serum transaminase. Alternatively, because the formation, intestinal absorption, and hepatic transport and metabolism of LC may vary, the elevations of the transaminase may be caused by an increased flux of LC through the liver. This variability also could explain the absence of an increased LC/CDC ratio in the bile of the inefficient sulfators, and of patients who developed elevations of the serum transaminase, and the lack of correlation between the total LC or sulfated LC in bile and the SGOT or SGPT. In addition, the poor reproducibility for the determination of LC in bile is of concern.

The fact that ursodeoxycholic acid also is converted to LC, albeit more slowly than CDC, yet appears to induce elevations of transaminase in humans only infrequently (37) militates against the hypothesis of hepatotoxicity mediated by LC. The question of hepatic injury by ursodeoxycholic acid is not yet settled, however, because in the Rhesus monkey, ursodeoxycholic acid and CDC had comparable hepatotoxic effects associated with similar increases in unsulfated LC in bile (38). As an alternative hypothesis for hepatic injury, increased levels of CDC in the serum may produce elevations of serum transaminase through a direct effect on permeability of the membrane of the hepatocyte. We, however, did not measure bile acids in the serum of our patients. The question of whether CDC produces hepatic injury by a direct mechanism has not been answered.

The value of a test can be illustrated by calculating, in addition to the sensitivity and specificity, the predictive value and efficiency of the test (39). In this study if the $S F$ is used as a predictor of elevations of serum transaminase in patients with gallstones, the sensitivity was $64 \%$, the specificity $93 \%$, the predictive value $78 \%$, and the efficiency $91 \%$. Values were similar when the SF as a predictor was analyzed only among patients receiving the high $(750 \mathrm{mg} / \mathrm{d})$ dose of CDC. Determination of the SF may be analogous to the determination of the ability of patients to acetylate for the prediction of isoniazid-induced hepatoxicity. The clinical value of determining SF in patients before therapy with CDC, however, has not been established. Whether determination of the SF can be applied to predict potential hepatoxicity of other therapeutic agents also remains to be determined.

\section{ACKNOWLEDGMENTS}

We acknowledge gratefully the expert technical assistance of Kim Goldman.

This work was supported in part by the National Cooperative Gallstone Study, National Institutes of Health contract 1-AM-3-2216 as an ancillary study AM-19032, and National Institutes of Health grant AM-15631.

\section{REFERENCES}

1. Thistle, J. L., A. F. Hofmann, B. J. Ott, and D. H. Stephens. 1978. Chemotherapy of gallstones: efficacy and safety. JAMA (J. Am. Med. Assoc.) 239: 1041-1046.

2. Koch, M., M. P. Giampieri, I. Lorenzini, A. M.Jezequel, and F. Orlandi. 1980. Effect of chenodeoxycholic acid on liver structure and function in man: a sterological and biochemical study. Digestion. 20: 8-21.

3. Danielsson, H., P. Eneroth, K. Hellstrom, S. Lindstedt, 
and J. Sjovall. 1963. On the turnover and excretory products of cholic and chenodeoxycholic acid in man. J. Biol. Chem. 238: 2299-2304.

4. Webster, K. H., M. C. Lancaster, and A. F. Hofmann. 1975. Influence of primary bile acid feeding on cholesterol metabolism and hepatic function in the Rhesus monkey. Mayo Clin. Proc. 50: 134-138.

5. Dryzka, H., G. Salen, F. G. Zaki, T. Chen, and E. H. Mosbach. 1976. Hepatic toxicity in the Rhesus monkey treated with chenodeoxycholic acid for six monthsBiochemical and ultrastructural studies. Gastroenterology. 70: 93-104.

6. Morrissey, K. P., C. K. McSherry, R. L. Swarm, W. H. Neiman, and J. E. Dietrick. 1975. Toxicity of chenodeoxycholic acid in the non-human primate. Surgery (St. Louis). 77: 851-860.

7. Palmer, R. H. 1972. Bile acids, liver injury and liver disease. Arch. Intern. Med. 130: 606-617.

8. Salen, G., H. Dryska, T. Chen, W. H. Saltzman, and E. H. Mosbach. 1975. Prevention of chenodeoxycholic acid toxicity with lincomycin. Lancet. I: 1082.

9. Cowen, A. E., M. G. Korman, A. F. Hofmann, O. W. Cass, and S. B. Cliffin. 1975. Metabolism of lithocholate in healthy man. 2. Enterohepatic circulation. Gastroenterology. 69: 67-76.

10. Loof, L., and B. O. Wengle. 1978. Enzymatic sulfation of bile salts in human liver (In vitro hepatic sulfation enzyme). Biochimica. et Biophys. Acta. 530: 451-460.

11. Palmer, R. H., and M. G. Bolt. 1971. Bile acid sulfates: synthesis of lithocholic acid sulfate and their identification in human bile. J. Lipid Res. 12: 671-679.

12. Schwenk, M., A. F. Hofmann, G. L. Carlson, J. A. Carter, F. Coulston, and H. Greum. 1978. Bile acid conjugation in chimpanzees. Arch. Toxicol. 40: 109-118.

13. Gadacz, T. R., R. N. Allan, E. Mack, and A. F. Hofmann. 1976. Impaired lithocholate sulfation in the Rhesus monkey: possible mechanism for chenodeoxycholate toxicity. Gastroenterology. 70: 1125-1129.

14. Palmer, R. H., C. K. McSherry, and P. S. May. 1977. Lithocholate metabolism in baboons ingesting chenodeoxycholic acid. Gastroenterology. 72: 1173 (Abstr.).

15. Allan, R. N., J. L. Thistle, and A. F. Hofmann. 1976. Lithocholate metabolism during chenotherapy for gallstone dissolution. 2. Absorption and sulfation. Gut. 17: 413-419.

16. Stiehl, A., P. Czygan, W. Frohling, M. Liersch, and B. Kommerell. 1977. Sulfation of bile acids. In: Liver and Bile. L. Bianchi, W. Gerok, and K. Sickinger, editors. MTP Press Ltd., Lancaster, England.

17. Allan, R. N., J. L. Thistle, A. F. Hofmann, J. A. Carter, and P. Y. S. Yu. 1976. Lithocholate metabolism during chenotherapy for gallstone dissolution. 1. Serum levels of sulfated and unsulfated lithocholates. Gut. 17: 405-412.

18. Cowen, A. E., M. G. Korman, A. F. Hofmann, and P. J. Thomas. 1975. Metabolism of lithocholate in healthy man. Plasma disappearance of radioactivity after intravenous injection of labeled lithocholate and its derivatives. Gastroenterology. 69: 77-82.

19. Hofmann, A. F., and P. Paumgartner. 1975. Chenodeoxycholic acid therapy of gallstones. Progress report. F. K. Schattauer Co., New York.

20. Lachin, J. M., J. W. Marks, and L. J. Schoenfield. 1981. Design and methodological considerations in the $\mathrm{Na}$ tional Cooperative Gallstone Study: a multi-center clinical trial. Controlled Clinical Trials. 2: 177-229.

21. Hofmann, A. F. 1964. Thin layer chromatography of bile acids and their derivatives. In New Biochemical
Separations. A. T. James and L. J. Morris, editors. D. Van Nostrand Company, New York. 261-282.

22. Fieser, L. F. 1948. Naphthoquinone antimalarials. XVI. Walter Soluble derivatives of alcoholic and unsaturated compounds. J. Am. Chem. Soc. 70: 3232-3237.

23. Grundy, S. M., and A. L. Metzger. 1972. A physiologic method for estimation of hepatic secretion of biliary lipids in man. Gastroenterology. 62: 1200-1217.

24. Van Berge Henengouwen, G. P., R. N. Allan, A. F. Hofmann, and P. Y. S. Yu. 1977. A facile hydrolysissolvolysis procedure for conjugated bile acid sulfates. J. Lipid Res. 18: 118-122.

25. Hegardt, F. G., and H. Dam. 1971. The solubility of cholesterol in aqueous solution of bile salt and lecithin. Z. Ernaehrungswiss. 10: 239-243.

26. Thomas, P. J., and A. F. Hofmann. 1973. A simple calculation of the lithogenic index of bile: expressing biliary lipid composition on rectangular coordinates. Gastroenterology. 65: 698-700.

27. Van Berge Henengouwen, G. P., and A. F. Hofmann. 1978. Nocturnal gallbladder storage and emptying in gallstone patients and healthy subjects. Gastroenterology. 75: 879-885.

28. Loof, L., and B. Wengle. 1979. Enzymatic sulfation of bile salts in man. Scand. J. Gastroenterol. 14: 513-519.

29. Bremelgaard, A., and L. Pedersen. 1976. Bile acids in bile during long term chenodeoxycholic acid treatment. Scand. J. Gastro. 11: 161-165.

30. Stiehl, A., R. Raedsch, and B. Kommerell. 1975. Increased sulfation of lithocholate in patients with cholesterol gallstones during chenodeoxycholate treatment. Digestion. 12: 105-110.

31. Gerolami, A., H. Sarles, and R. Brette. 1977. Controlled trial of chenodeoxycholic therapy for radiolucent gallstones. A multicenter study. Digestion. 16: 299-307.

32. Low-Beer, T. S., M. P. Tyor, and L. Lack. 1969. Effects of sulfation of taurolithocholic and glycolithocholic acids on their intestinal transport. Gastroenterology. 56: 721-726.

33. Palmer, R. H. 1971. Bile acid sulfates. II. The formation, metabolism and excretion of lithocholic acid sulfates in the rat. J. Lipid Res. 12: 680-687.

34. DeWitt, E. H., and L. Lack. 1980. Effects of sulfation patterns on intestinal transport of bile salt esters. J. Am. Physiol. Soc. 238: 34-39.

35. Carey, M. D., J. W. Shu-Fung, and J. B. Watkins. 1979. Solution properties of sulfated monhydroxy bile saltsrelative insolubility of the disodium salt of glycolithocholate sulfate. Elsevier North-Holland Biomedical Press.

36. Yousef, I. M., B. Tuchweber, R. J. Vonk, D. Masse, M. Audet, and C. C. Roy. 1981. Lithocholate cholestasissulfated glycolithocholate-induced intrahepatic cholestasis in rats. Gastroenterology. 80: 233-241.

37. Osuga, K. H., and the Tokyo Cooperative Gallstone Study Group. 1980. Efficacy and indications of ursodeoxycholic acid treatment for dissolving gallstones. Gastroenterology. 78: 542-548.

38. Sarva, R. P., H. Fromm, S. Farivar, R. F. Sembrat, H. Mendelow, H. Shinozuka, and S. K. Wolfson. 1980. Comparison of the effects between ursodeoxycholic and chenodeoxycholic acids on liver function and structure and on bile acid composition in the Rhesus monkey. Gastroenterology. 79: 629-636.

39. Galen, R. S., and S. R. Gambino. 1975. Beyond normality: the predictive value and efficiency of medical diagnoses. John Wiley \& Sons, Inc., New York. 